\title{
Fabrication and Measurement of Low-Stress Polyimide Membrane for High-Resolution Variable Optical Attenuator
}

\author{
Guo-Dung J. Su, Member, IEEE, Yu-Wei Yeh, Chen-Wei E. Chiu, Chao-Hu Li, and Tyng-Yow Chen
}

\begin{abstract}
We have fabricated low-stress polyimide membranes for use as high-resolution variable optical attenuator (VOA). Polyimide membranes are assembled with fiber collimators to perform 3-D beam spoiling that varies the optical power with 0.01-dB resolution. The measured residual stress and Young's modulus of the polyimide membrane are $3.2 \mathrm{MPa}$ and $15 \mathrm{GPa}$, respectively. The 41- $\mu \mathrm{m}$ deformation of the membrane is demonstrated by the electrostatic force with 170 volts. The 0.46-dB insertion loss and $>15-\mathrm{dB}$ dynamic range are achieved at $1550-\mathrm{nm}$ wavelength.
\end{abstract}

Index Terms-Large stroke, low stress, polyimide, variable optical attenuator (VOA).

\section{INTRODUCTION}

$\mathbf{E}$ RBIUM-doped fiber amplifiers have made long-haul wavelength-division multiplexing (WDM) transmission practical. Fiber amplifiers do permit multichannel transmission, but it is critical to maintain signal integrity for all channels after cascading a number of fiber amplifiers. Variable optical attenuator (VOA) is a device that manages signal power in fiber optic links by inducing variable loss. It is used to control the power level of optical signals between outputs of light sources and electrical-to-optical (E/O) converters. It can also be applied to test the linearity and dynamic range of photo sensors and photo detectors. Fiber variable optical attenuator (VOA) has been previously realized by means of liquid crystal, acousto-optic, and microelectromechanical systems (MEMS) devices [1]-[3]. Due to recent fast progress in micromachining fabrication, VOA made by MEMS technology shows high reliability and compact size, which make MEMS VOA promising for telecommunication applications [4]. It was proposed to implement VOA by the optical beam spoiling method [5] and 3-D beam spoiling was realized via a simple micromachined deformable mirror device that changes the focal length of a membrane [6]. The previous membrane approach, however, was made by brittle semiconductor materials. The stroke was generally limited to less than 10 $\mu \mathrm{m}$, and the highest attenuation was restricted to less than $5 \mathrm{~dB}$.

In this paper, we report a novel MEMS VOA consisting of a deformable polyimide thin film aligned with a fiber collimator in a free-space configuration. Due to the compliant structure of an organic thin film, the deformation of an organic thin film can

Manuscript received September 19, 2006; revised February 8, 2007. This work was supported in part by the National Science Foundation, Taiwan, R.O.C., under Contract 95-2221-E-002-275.

The authors are with the Graduate Institute of Electro-Optical Engineering and the Department of Electrical Engineering, National Taiwan University, Taipei 106 Taiwan (e-mail: gdjsu@cc.ee.ntu.edu.tw).

Digital Object Identifier 10.1109/JSTQE.2007.893270
TABLE I

LIST OF MATERIALS INDEXES OF THIN FILMS MADE By DIFFERENT MATERIALS

\begin{tabular}{lccc}
\hline \multicolumn{1}{c}{ Materials } & $\begin{array}{c}\text { Young's Modulus } \\
\boldsymbol{E}(\mathrm{Gpa})\end{array}$ & $\begin{array}{c}\text { Failure Strength } \\
\sigma_{\mathrm{f}}(\mathrm{Mpa})\end{array}$ & $\begin{array}{c}\text { Materials Index } \\
\boldsymbol{M}\end{array}$ \\
\hline Single Crystalline Silicon & 160 & 6600 & $4.13 \mathrm{E}-02$ \\
Polysilicon & 120 & 2500 & $2.08 \mathrm{E}-02$ \\
Silicon Nitride & 220 & 5300 & $2.41 \mathrm{E}-02$ \\
Nickel & 170 & 195 & $1.15 \mathrm{E}-03$ \\
Aluminum & 75 & 165 & $2.20 \mathrm{E}-03$ \\
Polyimide & 8.5 & 580 & $6.82 \mathrm{E}-02$ \\
\hline
\end{tabular}

attenuate the light intensity $>15 \mathrm{~dB}$ with $0.01-\mathrm{dB}$ resolution at the wavelength of $1550 \mathrm{~nm}$. The deformation of the membrane is up to $41 \mu \mathrm{m}$ without permanent warps, and the membrane size is $6 \mathrm{~mm} \times 6 \mathrm{~mm}$ separated from a bottom electrode by $70 \mu \mathrm{m}$. First, we discuss the design principle of the device. In Sections II and III, we describe fabrication processes and experimental measurements. Finally, we summarize the paper in Section IV.

\section{MAterial SELECTION AND FABRICATION}

Microfabricated VOA by using deformable membrane was demonstrated earlier [6]. The attenuation range, however, was limited by small strokes due to material properties. In principle, the maximum displacement of an actuator is determined by failure strength of the material (yield and fracture strength for ductile and brittle materials, respectively). In order to compare different materials, a materials index $M$ is defined as [7]

$$
M=\frac{\sigma_{f}}{E}
$$

where $\sigma_{f}$ is the failure strength and $E$ is Young's modulus. Materials of large values of $M$ are good candidates for high stroke actuators. Mechanical properties of popular materials, including silicon, nitride, and aluminum, are listed in Table I. It is clear that the materials index of polyimide is the highest among listed materials and is a reasonable choice for large stroke applications.

In addition to the effects of Young's modulus and failure strength, one must also take residual stress of thin films into consideration. According to the load-deflection effect, we can obtain the relationship between pressure and deflection as [8]

$$
p(h)=C_{1} \frac{1 \sigma}{a^{2}} h+C_{2}(v) \frac{t E}{a^{4}} h^{3}
$$

where $p$ is the applied pressure, $h$ is the center displacement, $a$ is one half of the membrane's edge length, $t$ is the thickness of a membrane, $E$ is Young's modulus, $\sigma$ is the residual stress, and 
a)
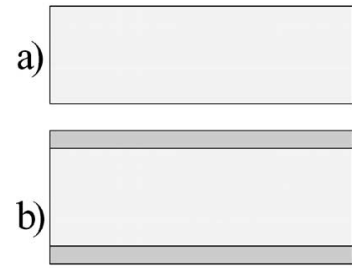

e)
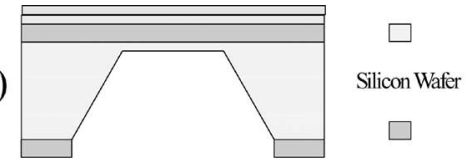

f)

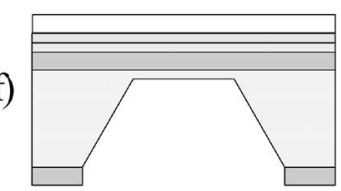

Silicon Dioxide

c)

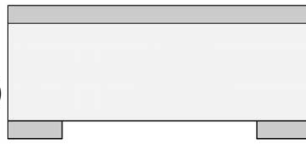

d)
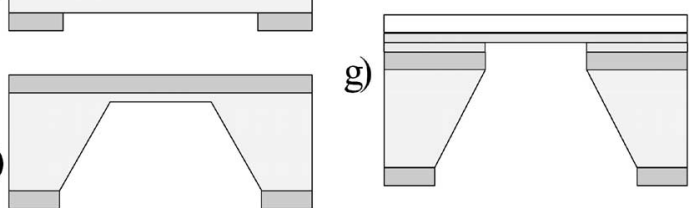

Fig. 1. Schematic drawing of fabrication process.

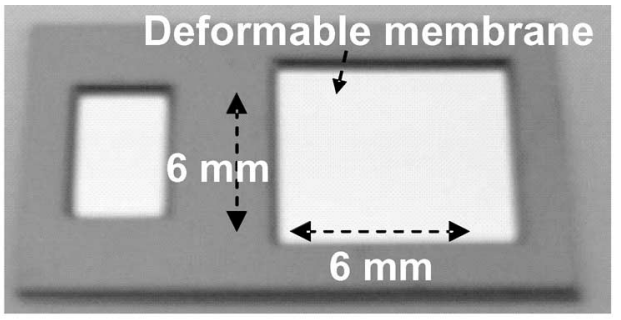

(a)

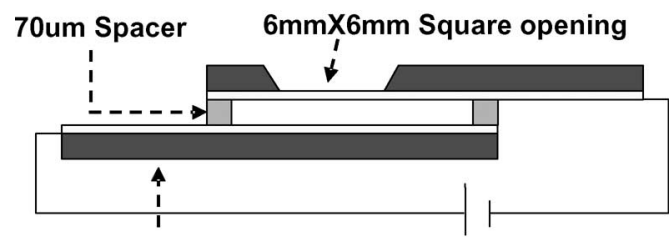

Bottom electrode

(b) $v$ is Poisson's ratio. $C_{1}$ and $C_{2}$ are numerical constants, which are determined by curve fitting to a load-deflection experiment. We can see that the residual stress is another effective factor in addition to Young's modulus, which determine the needed force. Therefore, if we want to get large strokes with reasonable low actuation force, we must lower the residual stress as well. The most frequently used low-stress membrane materials are single crystal silicon and silicon nitride [9]. Their residual stress are 3-7 MPa and 50-100 MPa, respectively. Young's modulus is $160 \mathrm{GPa}$ for silicon and $220 \mathrm{GPa}$ for silicon nitride. The numbers, however, are large to make deformable membranes with large strokes that can be actuated by electrostatic force. Ideally, we would like to have a membrane with residual stress as low as single crystal silicon, while Young's modulus is maintained one order of magnitude lower than the numbers earlier. As a result, we should look for other appropriate materials to be a membrane structure layer.

It is reported that the yield strain of an organic polymer is around 5\%, which far exceeds the breaking limit of semiconductor materials, and Young's modulus is about one order of magnitude lower than that of most inorganic materials [10]. Moreover, some polymers have excellent chemical resistance during wet etching and can be coated at room temperature either by spin coating or injection printing, which require less complicated fabrication processes as compared with standard semiconductor production lines. Based on those characters, organic polymer is an ideal candidate for MEMS deformable membrane applications. We selected a low stress polymer based on biphenyldianhydride/1,4-phenylenediamine backbone chemistry to be our membrane material.

The fabrication process of low stress polyimide membrane is illustrated in Fig. 1. Starting with a 4-in ( $\left.\begin{array}{lll}1 & 0 & 0\end{array}\right)$ silicon wafer, 300 -nm silicon dioxide was thermally grown on a wafer as a wet etching protection mask. A $6 \mathrm{~mm} \times 6 \mathrm{~mm}$ square opening was patterned on thermal oxide and etched by buffer HF at the backside of the silicon wafer, as shown in Fig. 1(c). The silicon wafer was then anisotropically etched in the solution of tetramethyl ammonium hydroxide (TMAH) at $90^{\circ} \mathrm{C}$, as the
Fig. 2. (a) Picture of a fabricated polyimide membrane. (b) Schematic drawing of an assembled device.

silicon etching rate and surface roughness are sensitive to the temperature of TMAH. The etching depth of the silicon was time-controlled to left about 30- $\mu \mathrm{m}$ silicon membranes before etching through the wafer. The $100-\mathrm{nm}$ aluminum was then deposited by e-beam evaporator on the front side of the silicon wafer with a $20-\mathrm{nm}$ chromium seed layer. A $2-\mu \mathrm{m}$ organic thin film was then spin-coated and baked at $350^{\circ} \mathrm{C}$ in an oven to dry solvents out in the step of Fig. 1(f). Finally, the remaining silicon was etched by deep reactive ion etching (DRIE) and stopped at thermal oxide layer. Thermal oxide and chromium were removed by pad etchant (Pad Etch 4 from Ashland) and Cr etchant (Cr-7T). Pad etchant, which contains acetic acid, ammonium fluoride, surface tension and water, could minimize damages to metal layers. An aluminum reflection layer was undamaged after the etching process and has a good reflectivity at optical communication wavelength [11]. Fig. 2(a) shows a photograph of the fabricated device. The surface of the polymer membrane is smooth due to the tensile residual stress of the polyimide and the surface roughness is measured to be around $20 \mathrm{~nm}$ by a white light interferometer. The schematic drawing in Fig. 2(b) shows a polyimide membrane is assembled with a bottom electrode to generate the electrostatic force for actuating a membrane. Experimental results are discussed in Section III.

\section{Stress Measurement And DeVice Characterization}

A load-deflection experiment [12] is applied to measure the residual stress and Young's modulus of a membrane. Equation (1) can be translated to (2)

$$
p^{\prime}\left(h^{\prime}\right)=C_{1} \frac{t \sigma}{a^{2}}+C_{2}(v) \frac{t E}{a^{4}} h^{\prime}
$$

where $p^{\prime}=p / h$ and $h^{\prime}=h^{2}$. As a result, $p^{\prime}$ becomes a liner function that the slop is proportional to Young's modulus and the intercept is proportional to the residual stress. The measurement was done by using pneumatic force to pull down a membrane and 


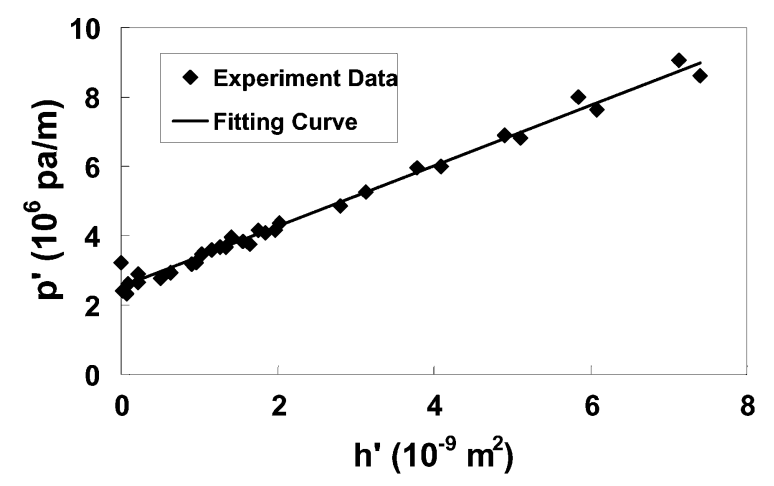

Fig. 3. Experimental results of residual stress and Young's modulus.

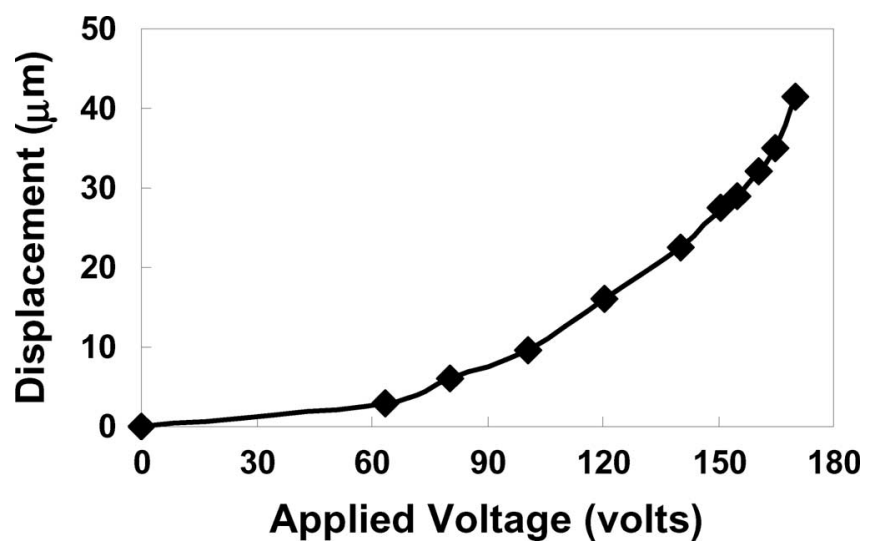

Fig. 4. Experimental results of center displacement versus applied voltage.

experimental results are shown in Fig. 3. The length of the tested membrane is $6 \mathrm{~mm}$ and total thickness is $2.35 \mu \mathrm{m}$. According to the fitting curve, we concluded that residual stress is $3.2 \mathrm{MPa}$ and Young's modulus is $15 \mathrm{GPa}$. We believe that the residual stress can be lowered by optimizing substrate condition because the multilayer coefficient of thermal expansion (CTE) mismatch would alter the stress during a polymer curing step [13]. The polymer membrane is actuated by the electrostatic force. The schematic drawing in Fig. 2(b) shows the experiment setup. A bottom electrode and a membrane are separated by a $70-\mu \mathrm{m}$ spacer. The center displacement versus applied voltage is shown in Fig. 4. The maximum deformation toward bottom electrode is about $41 \mu \mathrm{m}$ as applying $170 \mathrm{~V}$, and its equivalent radius of curvature is approximately $110 \mathrm{~mm}$. This is about one order improvement over the popular deformable mirrors from OKO Technology, Holland, the membranes of which are made with silicon nitride [14]. The snap down effect of a membrane is observed when the stroke of a membrane is slightly larger than half of the gap spacing. There is no permanent damage to the membrane when it is snapped down because the yield strength of a polyimide membrane can survive such deflection.

A deformable membrane has a lot of applications, such as adaptive optics [15], and $1 \times \mathrm{N}$ optical switches [16], etc. In this paper, we made use of fabricated membranes as high-resolution variable optical attenuators. The working principle is based on the phenomenon that the focal length of a deformable mirror is altered when a membrane is changed from a flat surface to

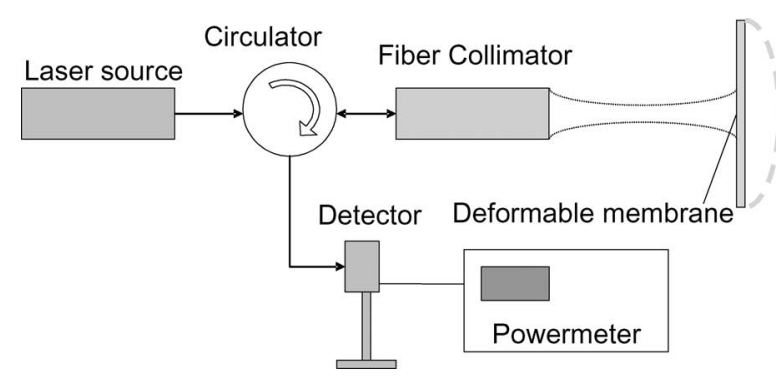

Fig. 5. Schematic drawing of a deformable membrane VOA.

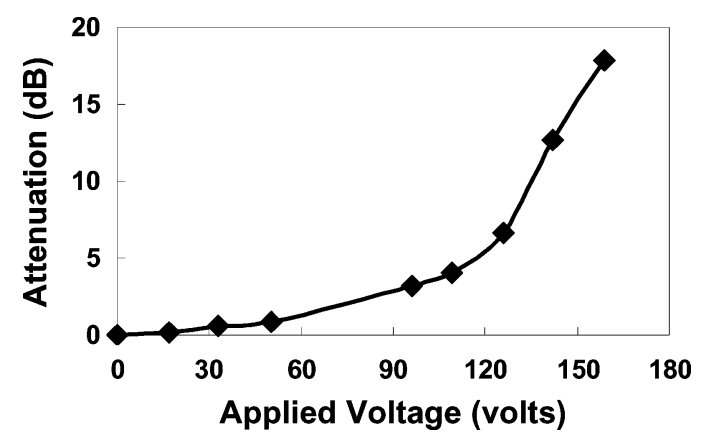

Fig. 6. Attenuation versus applied voltage.

a concave shape. This variable focusing effect can be used to change the working distance of a self-imaging fiber collimator, as shown in Fig. 5. In this design, a single collimator lens is used as the input and output port of the VOA via an optical circulator. The total insertion loss of the device is $3.61 \mathrm{~dB}$, including the circulator and the single collimator lens. The circulator shows $1.56 \mathrm{~dB}$ and $1.49 \mathrm{~dB}$ losses from port 1 to port 2 and port 2 to port 3 , respectively. The single collimator lens introduces additional 0.1-dB loss. Therefore, the polyimide membrane accounts for the remaining $0.46-\mathrm{dB}$ loss, which is in part caused by surface roughness of the membrane. The expanded optical beam with $2.2-\mathrm{mm}$ diameter is reflected by the membrane mirror and is coupled back into the collimator. The path length for the return signal is kept equal to the working distance of the lens. The focusing characteristic of the deformable mirror is used to change this working distance for an optical beam, hence, introducing a coupling loss. Fig. 6 shows the experimental data of optical attenuation versus applied voltage. The maximum optical attenuation is around $18 \mathrm{~dB}$ at $170 \mathrm{~V}$. Compared with a similar silicon nitride membrane design [6], the attenuation range is improved from less than 5 to about $18 \mathrm{~dB}$. The average slope of attenuation is about $0.1 \mathrm{~dB} / \mathrm{V}$. In theory, fine adjustment of $0.005 \mathrm{~dB}$ could be achieved by utilizing a dc power supply with $50-\mathrm{mV}$ resolution. However, in the lab experiment, the best resolution we observed is $0.01 \mathrm{~dB}$ because the minimum digit of our power meter is $0.01 \mathrm{~dB}$.

To measure the response time of the membrane, an openloop square wave was applied directly to the device. When the electrical signal was switched on, the membrane was deformed to its maximum stroke and the optical signal was dropped accordingly. The response time was $14 \mathrm{~ms}$ from zero attenuation to higher attenuation range, as shown in Fig. 7. Regarding the 


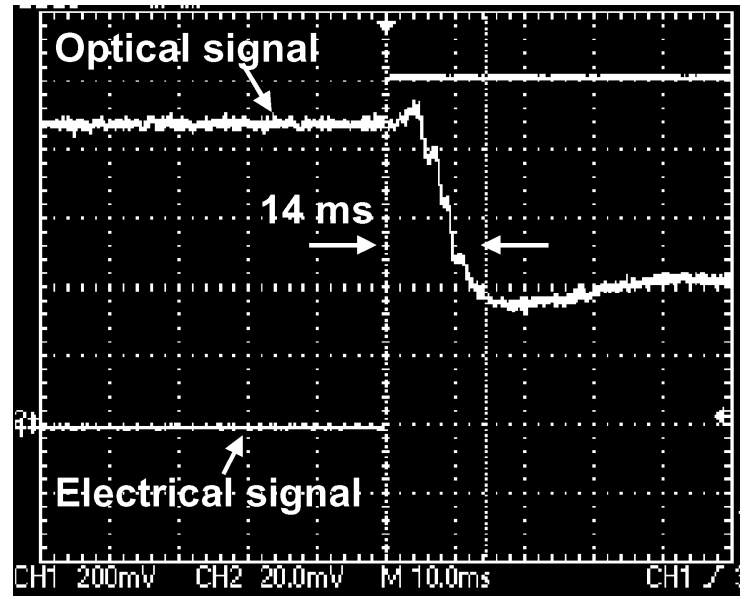

Fig. 7. Response time of VOA.

long-term reliability, we suspect that moisture absorption and oxygen might break down the chemical links inside organic materials, as reported in other organic light emitting diode (OLED) research works [17]. As a result, appropriate hermetic packaging and surface treatment [18] are needed to ensure the reliability of a polyimide membrane.

\section{CONCLUSION}

A polyimide-based deformable membrane was successfully fabricated. Its surface roughness was measured about $20 \mathrm{~nm}$ and the insertion loss caused by the membrane is $0.46 \mathrm{~dB}$. The load-deflection experiment result shows significantly low residual stress of 3.2 $\mathrm{MPa}$ and Young's modulus of $15 \mathrm{GPa}$. According to these material properties, the displacement as large as $41 \mu \mathrm{m}$ is achieved without any crack. Since the deformable range is larger, it can be potentially applied to many other optical applications.

\section{REFERENCES}

[1] K. Hirabayashi, M. Wada, and C. Amano, "Optical-fiber variable attenuator arrays using polymer-network liquid crystal," IEEE Photon. Technol. Lett., vol. 13, no. 5, pp. 487-489, May 2001.

[2] M. J. Mughal and N. A. Riza, "Compact acousto-optic high speed variable attenuator for high power applications," IEEE Photon. Technol. Lett., vol. 14, no. 4, pp. 510-512, 2002.

[3] K. Isamoto, K. Kato, A. Morosawa, C. Chong, H. Fujita, and H. Toshihyoshi, "A 5-V operated MEMS variable optical attenuator by SOI bulk micromachining," IEEE J. Sel. Topics Quant. Electron., vol. 10, no. 3, pp. 570-578, May-Jun. 2004.

[4] A. Neukermans and R. Ramaswami, "MEMS technology for optical networking applications," IEEE Commun. Mag., vol. 39, no. 1, pp. 62-69, Jan. 2001.

[5] P. Colbourne, J. Obhi, and N. Teitelbaum, "Variable optical attenuator," U.S. Patent 5915 063, Jun. 22, 1999.

[6] R. J. MacDonald, L. Yang, P. Lonjoux, P. Colbourne, and M. Mohiuddin, "Fast attenuator," U.S. Patent 6462 858, Nov. 28, 2002.

[7] V. Srikar and S. Spearing, "Materials selection for microfabricated electrostatic actuators," Sens. Actuators A, vol. 102, pp. 279-285, 2003.

[8] D. Maier-Schneider, J. Maibach, and E. Obermeier, "New analytical solution for the load-deflection of square membranes," J. Microelectromech. Syst., vol. 4, no. 4, pp. 238-241, 1995.

[9] P. Kurczynski, H. Dyson, B. Sadoulet, J. Bower, W. Lai, W. Mansfield, and J. Taylor, "Fabrication and measurement of low-stress membrane mirrors for adaptive optics," Appl. Opt., vol. 43, no. 18, pp. 3573-3580, 2004.
[10] P. Hsu, M. Huang, S. Wagner, Z. Suo, and J. Sturn, "Plastic deformation of thin foil substrates with amorphous silicon islands into spherical shape," Mater. Res. Soc. Proc., vol. 621, pp. Q8.6.1-Q8.6.6, 2000.

[11] J. M. Bennett and E. J. Ashley, "Infrared Reflectance and Emittance of Silver and Gold Evaporated in Ultrahigh Vacuum," Appl. Opt., vol. 4, no. 2, pp. 221-224, 1965.

[12] O. Tabata, K. Kawahata, S. Sugiyama, and I. Igarashi, "Mechanical property measurements of thin films using load-deflection of composite rectangular films," Sens. Actuators, vol. 20, pp. 135-141, 1989.

[13] T. Harder, T. J. Yao, Q. He, C. Y. Shih, and Y. C. Tai, "Residual Stress in thin-film Parylene-C," in Proc. 15th IEEE Int. Conf. Micro Electro Mech. Syst., pp. 435-438, 20-24, 2002.

[14] G. Vdovin and P. Sarro, "Flexible mirror micromachined in silicon," Appl. Opt., vol. 34, no. 16, pp. 2968-2972, 1995.

[15] L. Zhu, P. Sun, D. Bartsch, W. Freeman, and Y. Fainman, "Adaptive control of a micromachined continuous-membrane deformable mirror for aberration compensation," Appl. Opt., vol. 38, no. 1, pp. 168-176, 1999.

[16] Y. Peter, F. Gonte, H. Herzig, and R. Dandliker, "Micro-optical fiber switch for a large number of interconnects using a deformable mirror," IEEE Photon. Technol. Lett., vol. 14, no. 3, pp. 301-303, Mar. 2002.

[17] P. Burrows, V. Bulovic, S. Forrest, L. Sapochak, D. McCarty, and M. Thompson, "Reliability and degradation of organic light emitting devices," Appl. Phys. Lett., vol. 65, no. 23, pp. 2922-2924, 1994.

[18] J. Kim, M. Lebbai, Y. Lam, P. Hung, and R. Woo, "Effects of moisture and temperature ageing on reliability of interfacial adhesion with black copper oxide substrate," J. Adhesion Sci. Technol., vol. 19, no. 6, pp. 427-444, 2005.

Guo-Dung J. Su received the B.S. degree from National Taiwan University (NTU), Taipei, Taiwan, in 1994. He received the M.S. and Ph.D. degrees in electrical engineering from the University of California, Los Angeles, in 1998 and 2001, respectively. His doctoral thesis was related to MEMS scanners with flat mirror surfaces for active optical alignment and micromirror arrays for adaptive optics applications.

Currently, he is an Assistant Professor with the Graduate Institute of ElectroOptical Engineering and Department of Electrical Engineering, NTU. His research interests include MEMS devices for optical communications, compact optical imaging systems, and surface plasmon phenomenon on nano particles.

Yu-Wei Yeh received the B.S. degree in physics from the National Cheng-Kung University, Tainan, Taiwan and the Master's degree from the Graduate Institute of Electro-Optical Engineering, National Taiwan University, Taipei, Taiwan, in 2004 and 2006, respectively.

His research interest includes MEMS-based optical components, especially in variable optical attenuator for fiber communication.

Chen-Wei Chiu received the B.S. degree in mechanical engineering from the National Taiwan University (NTU), Taipei, Taiwan and the M.S. degree from the University of California, Los Angeles, in 1994 and 1998, respectively.

$\mathrm{He}$ is a cofounder of Umachines and still contributes his expertise in the optical MEMS field through collaboration with NTU.

Chao-Hu Li received the B.S. degree in material science from the National Taiwan University, Taipei, Taiwan, in 2006, where he is currently working toward the Master's degree from the Graduate Institute of Electro-Optical Engineering of the same university.

His research interest includes polymer thin films and their applications.

Tyng-Yow Chen received the B.S. degree in electrical engineering from National Taiwan University, Taipei, Taiwan, in 2005, where he is working toward the Master's degree.

Currently, he is developing large-stroke deformable mirrors. 